\title{
Performance Prediction and Reverse Design of a Glass Composites Pultrusion Process Based on RBF
}

\author{
Junming Liu ${ }^{\mathrm{a}}$, Hanwu Liu ${ }^{\mathrm{b}}$, Shuai $\mathrm{Luo}^{\mathrm{c}}$, Zhiwen Guo ${ }^{\mathrm{d}}$ \\ School of Mechanical and Electrical Engineering, North China Institute of Science and Technology, \\ Sanhe, Hebei, 065201, China

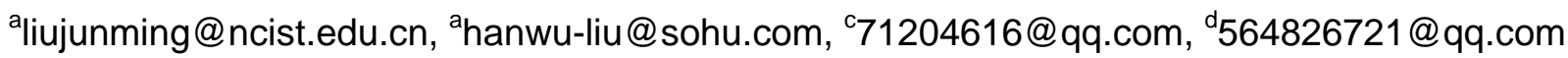

Keywords: Glass Composites; Curing Degree; Mould Temperature; Performance Forecast

\begin{abstract}
Most glass composites products in engineering are manufactured by a pultrusion process. Because the pultrusion process is affected by many factors, it is not easy to control the performance of products. In order to obtain good performance and meet the requirements of glass composites products in specific parameters, artificial neural network technology was adopted to optimize and design the pultrusion process. The results showed that the curing degree of products increased gradually with the preheated mould temperature. During the reverse design of glass composites products, it was found that when the spread is 0.3 , the measured temperature values of every stage based on curing degree were in good agreement with the forecasting values, which proved the correctness of the reverse design. The research method in this paper provides important theoretical basis and technical support for similar products.
\end{abstract}

\section{Introduction}

Glass reinforced plastic is commonly known as reinforced composite plastic, also known as Glass Fiber Reinforced Plastic (GFRP) [1]. It is made of synthetic resin as an adhesive and glass as a reinforcing material. Composite materials are a combination of different bases or reinforcing materials, such as organic polymer material, metal and inorganic non-metallic, in various processes. In addition to retaining the main features of component materials, composite materials can also obtain unique properties that component materials do not have through composite effect. With the development of the times, cement or non-metallic based composite materials have been gradually replaced by GFRP. Most cable protection tubes made of GFRP have better corrosion resistance and span with a smooth surface which is beneficial for cleaning [2].

Pultrusion is a typical process for manufacturing GFRP continuously and economically with many features, such as high production efficiency, good reproducibility, arbitrary length, raw material saving, energy saving. In pultrusion process of GFRP, temperature is one of the key factors affecting the forming process [3] [4]. When the curing degree meets the requirements, if the temperature is the optimum value, the energy consumption can be reduced and the costs can be saved with the competitiveness of the products increasing.

In this paper, the process plan of a GFRP pipe was firstly determined by analyzing its composition, studying its properties and the pultrusion process. According to the material properties, the temperature of each stage was analyzed and calculated. Then the network model of the temperature and curing degree was established, as based on the Radial Basis Function artificial neural network (RBF) by using MATLAB [5] [6]. After training, the network model could be used to predict the temperature of each stage and then get the optimum temperature, which could greatly cut down on the amount of time spent on experiments, reduce production costs, and improve the success rate of pultrusion forming.

\section{Training and Prediction of RBF Network}

The size of the GFRP pipe is: $\mathrm{D}=22 \mathrm{~mm}, \mathrm{~d}=6 \mathrm{~mm}$, the total length of pipe in one production of $1000 \mathrm{~mm}$, pultrusion rate of $20 \mathrm{~m} / \mathrm{h}$. This part is mainly installed in the crossing area of the communication cable and power line to prevent a short circuit caused by the break of power line which may cause the communication cable and wire rope charged and then to protect cables, 
switches, core boards and even the machine from being burned. Also this part can isolate magnetic field interference of the power line.

1) Training Algorithms and Measurement Indicators

In general, there are two methods to train and test artificial neural network models. One is 'Leave-one-out', the other is 'dichotomy'. By comparison, 'Leave-one-out' can achieve a high forecasting accuracy and get the best, reliable forecasting accuracy, though it has a high workload and is time-consuming. In this paper, 'Leave-one-out' is used for training and testing RBF network model. Specifically, based on using GFRP temperature of each stage as input variables, the curing degree as output variables and then building a network model of three inputs and one output, the curing degree of GFRP can be predicted after repeated training. Afterwards, the temperature can be determined according to the curing degree through reverse design, which can reduce experimental costs with a high precision and high efficiency. 7 set of data are used to train the network model and by selecting different spread values, the optimal model can be achieved.

For the qualitative and quantitative measurement of the forecast performance of RBF, scatter plots and statistical indicators are selected. The three statistical indicators selected for quantitative measuring forecast performance are Mean Square Error (MSE), Mean Square Relative Error (MSRE) and Value of Fitness (VOF), their formulas are shown respectively:

$$
\begin{gathered}
M S E=\sqrt{\frac{\sum_{i=1}^{N}\left(X_{1 i}-X_{2 i}\right)^{2}}{N} M S R E=\sqrt{\frac{\sum_{i=1}^{N}\left(\frac{\left.X_{1 i}-X_{2 i}\right)^{2}}{X_{2 i}}\right.}{N}}} \\
V O F=1+\frac{\sum_{i=1}^{N}\left(X_{1 i}-\bar{X}_{1 i}\right)\left(X_{2 i}-\bar{X}_{2 i}\right)}{\sqrt{\left(\sum_{i=1}^{N} X^{2}{ }_{1 i}-N \bar{X}^{2}{ }_{1 i}\right)\left(\sum_{i=1}^{N} X^{2}{ }_{2 i}-N \bar{X}^{2}{ }_{2 i}\right)}}
\end{gathered}
$$

Where: $X_{1 i}$ is the measured value, $X_{2 i}$ is the predicted value, $N$ is the sample size.

When the predicted value and the measured value get closer, which is when the forecast performance becomes better, MSE and MSRE get smaller and approach to 0, the VOF gets bigger and approaches to 2. When MSE and MSRE get bigger and farther from 0, the VOF get smaller and farther from 2, the forecast performance becomes worse.

2) Training Results

Table 1. Training and optimization of data (GFRP temperature and curing degree)

\begin{tabular}{ccccc}
\hline \multirow{2}{*}{ Number } & \multicolumn{3}{c}{ Temperature $\left({ }^{\circ} \mathrm{C}\right)$} & \multirow{2}{*}{ Curing degree $(\%)$} \\
\cline { 2 - 4 } & First stage & Second stage & Third stage & 90.52 \\
2 & 119 & 149 & 143 & 90.47 \\
3 & 121 & 147 & 145 & 91.03 \\
4 & 123 & 150 & 148 & 91.94 \\
5 & 126 & 156 & 153 & 91.64 \\
6 & 128 & 153 & 150 & 92.61 \\
7 & 133 & 161 & 152 & 93.07 \\
8 & 135 & 165 & 155 & 93.43 \\
9 & 138 & 168 & 157 & 93.69 \\
\hline
\end{tabular}

Structure and Training Elements of RBF Network. The structure of an RBF Network model includes the number of layers in the model, the number of neurons in each layer, the type of the activation function and the spread value of the model, etc. Here the significance of spread value is explained. The model prediction accuracy is determined by the size of the spread value. A lower spread value means better forecasting accuracy but more time consumption, while higher spread value needs less time but means worse prediction accuracy. In order to ensure accuracy but also within a short time, it is necessary to adjust spread value constantly in the adjustment process so as to achieve the best combination of accuracy and time. The model training is selecting multiple sets of data to train the neural network, and selecting different spread values to determine the optimal model, which is ready for forecasting. After training the model, the structure of the network model 
has been adjusted and optimized. If a new variable is entered, the model can predict the result afterwards. As seen in table 1, 9 set of data have been selected to train, wherein the pultrusion speed is $20 \mathrm{~m} / \mathrm{h}$.

Influence of Spread Value. 7 set of data were randomly selected for training model, and group $1,2,3,5,6,7,9$ were selected in this training. Meanwhile the spread values were changed to observe the effect of different training. Fig.1 shows the training performance when the spread value is $0.4,0.5,0.6,0.7,0.8$, and 1 .
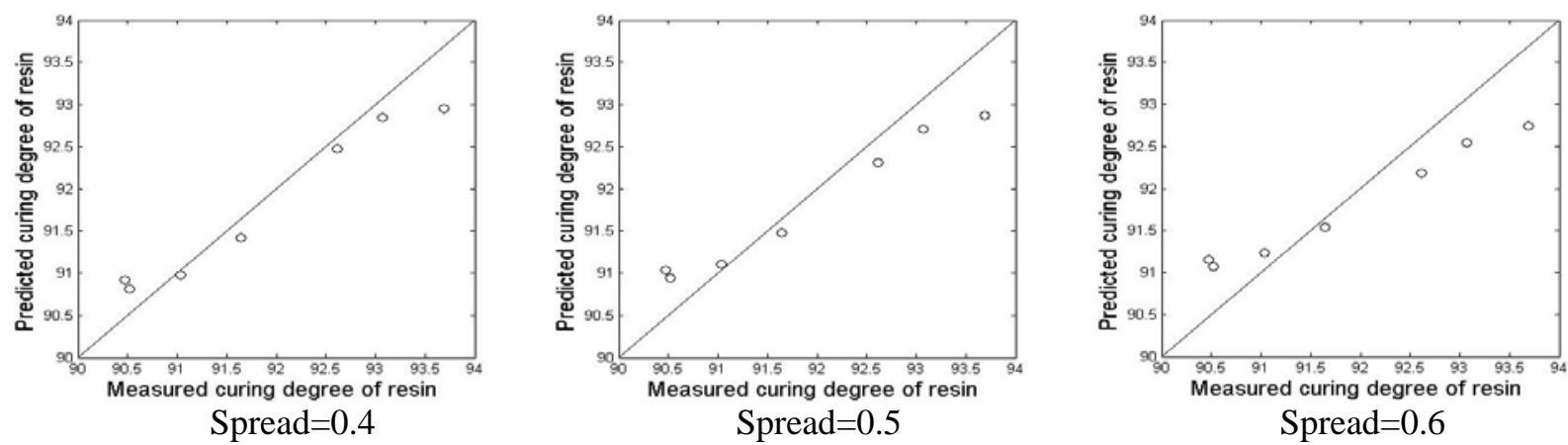

Spread $=0.5$

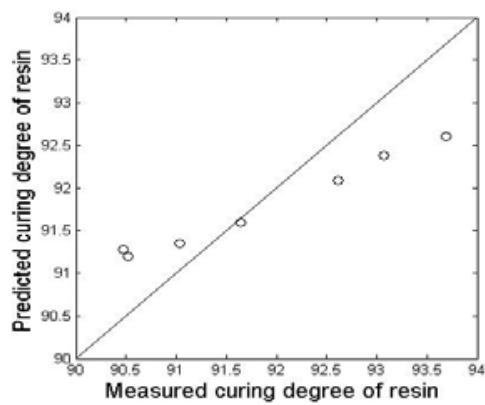

Spread $=0.7$

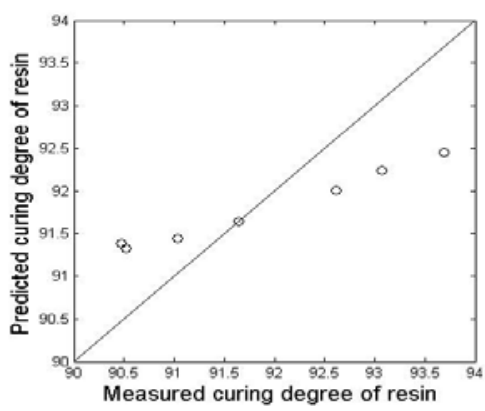

Spread $=0.8$
Spread $=0.6$

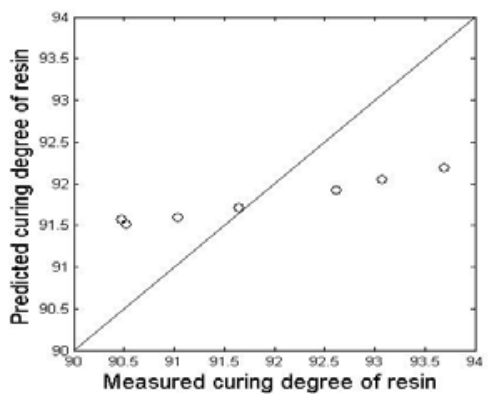

Spread=1

Fig. 1 Scatter plots with different Spread values

As can be seen from the Fig. 1, when the spread value is $0.4,0.5$ or 0.6 , the points on scatter plots distribute substantially along the $45^{\circ}$ diagonal. However, the distribution of points along the $45^{\circ}$ diagonal seems worse and worse with the spread values increasing. It can be seen that when the spread value reaches 1 , the scatter distribution is substantially horizontal which indicates more training errors and a less effective training result. By synthesizing analyzing the scatter plots with different spread values, the range of optimal spread value in the RBF training model can be qualitatively determined, and some of the changes between predicted and measured values in the graph can be easily read out. The training performance of the artificial neural network model can be directly qualitatively described through the scatter plot, but quantitative description must rely on statistical indicators.

Statistical Indicators. From Fig. 1, it can be seen apparently that with the spread value increasing, the prediction error decreases firstly and then increases, which means that the prediction accuracy increases firstly and then decreases as the spread value increases. Different statistical indicators can be obtained by taking training results based on different spread values into the training program. Table 2 shows mean square error, mean square relative error, and the value of fitness.

Table 2. MSE, MSRE, and VOF

\begin{tabular}{cccc}
\hline Spread value & MSE & MSRE & VOF \\
\hline 0.4 & 0.3689 & 0.0040 & 1.9834 \\
0.5 & 0.4515 & 0.0049 & 1.9877 \\
0.6 & 0.5591 & 0.0061 & 1.9909 \\
0.7 & 0.6728 & 0.0073 & 1.9925 \\
0.8 & 0.7786 & 0.0084 & 1.9929 \\
1 & 0.9475 & 0.0103 & 1.9918 \\
\hline
\end{tabular}


By the table 2, with the increasing spread values, mean square error and relative error are growing, indicating the greater the deviation of the predicted value and the measured value. When the spread value is of 0.4 , the mean square error and relative error relative to the other groups are relatively small, and the fitted values relatively close to 2 , it was decided to optimize the use of this spread is 0.4 forecast.

\section{Forecasting and Impact of Heating Temperature on Curing Degree}

After training and testing of the artificial neural network model, the structure has been optimized, with the best, most reliable prediction performance. The best spread value is 0.4 , and on this condition the RBF model can be used for forecasting curing degree on the temperature of different stages. The samples were in two groups, numbered 4 and 8. Comparisons of measured and predicted results of temperature on curing degree are shown in Table 3.

Inputting measured and predicted values into program for calculation of statistical indicators, the results are as follows: $\mathrm{MSE}=0.2007 ; \mathrm{MSRE}=0.0022$; VOF $=1.9987$. It can be clearly seen from statistical indicators that Mean Square Relative Error (MSRE) and Mean Square Error (MSE) are very close to zero, and the Value Of Fitness (VOF) is very close to 2. So the predicted results meet the ideal requirements, and this RBF model can be used in the pultrusion production of GFRP pipes for optimizing the forming process.

Table 3 The measured and predicted values

\begin{tabular}{lll}
\hline Number & \multicolumn{1}{c}{4} & 8 \\
\hline Measured value & 91.94 & 93.43 \\
Predicted value & 91.7537 & 93.2159 \\
\hline
\end{tabular}

Fig. 2 shows the influence of temperature on curing degree at different stages. As can be seen, with the temperature increasing, the curing degree also gradually increased with nonlinear relationship between the two. In actual production, the temperature is generally not too high considering the production efficiency and energy saving. Under the condition of obtaining appropriate performance, the temperature should not be too high.

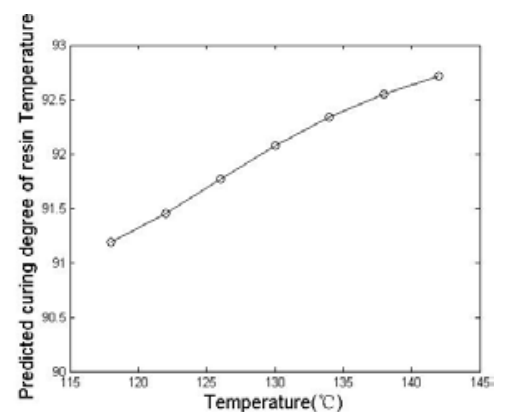

(a) The first stage

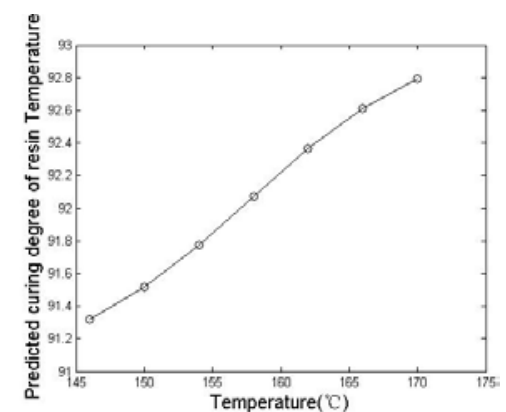

(b) The second stage

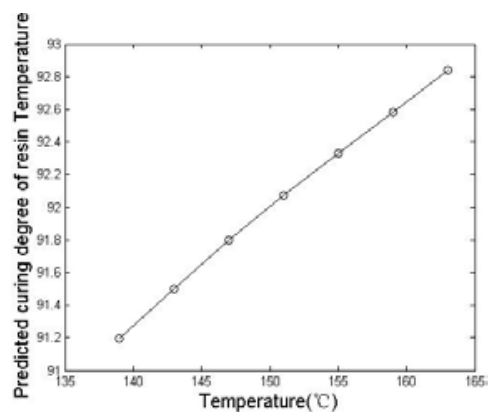

(c) The third stage

Fig. 2 Influence of temperature on curing degree

\section{Reverse Design of Process Parameters Based on RBF Network}

According to the curing degree, reverse design is adopted to design and optimize the temperature of each stage for achieving the best predicted value. Similarly with predicting the curing degree, the artificial neural network model need training before using, called the 'learning phase of model'. The purpose is to adjust the structure of the artificial neural network model, reflect the intrinsic relationship between the input and output variables of training samples, and get the model that has the best prediction performance. Spread values $0.3,0.4,0.5,0.6$ were selected to train the neural network model. After training, it was found that when the spread value is 0.3 , the mean square error and mean square relative error are relatively small, and the value of fitness is close to 2 with the best performance. Fig. 3 shows reverse design of curing degree on temperature when Spread $=0.3$. 


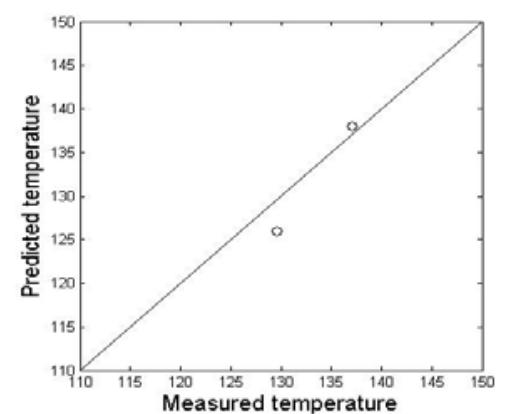

(a) The first stage

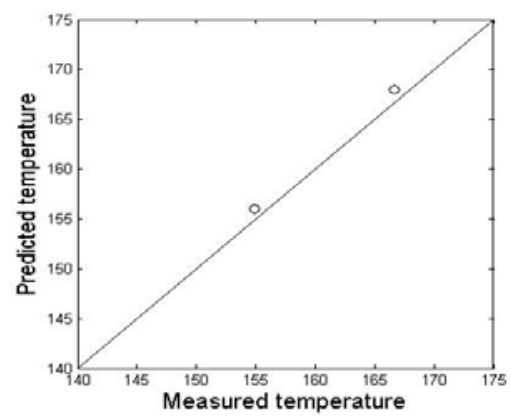

(b) The second stage

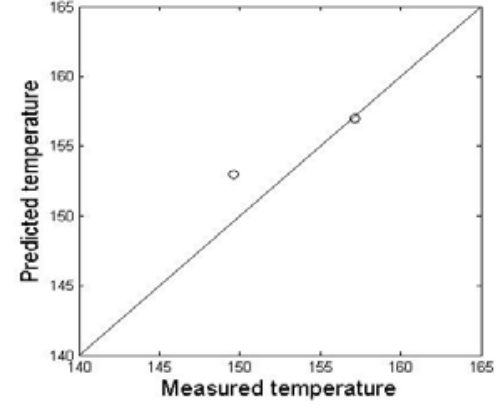

(c) The third stage

Fig. 3 Scatter plot of reverse design of curing degree on temperature when Spread is 0.3

As it can be seen above, when the spread value is 0.3 , the forecast temperature data for each stage almost focuses on the 45 degree line, indicating the predicted temperature of each stage by curing degree has almost no error with the measured temperature. Due to the noise and quality problem of samples, the mean square error and mean square relative error do not equal zero, but it has basically reached the requirement. Meanwhile the value of fitness is close to 2, indicating that the reverse design of temperature for each stage is quite accurate.

\section{Conclusion}

According to the pultrusion curing degree of GFRP pipes, a neural network model was establish and after training it was used for predicting the relationship between temperatures and curing degrees at different stages of pultrusion. First, to meet relevant requirements, the spread value based on the training results should be modified. Then after training and testing, the sample could be predicted and the forecasting results were described by statistical indicators. The results show that:

1) By using RBF, not only the curing degree can be predicted according to the temperature of each stage, but also the temperature can be forecasted by curing degree with reverse design.

2) After forecasting, the influence of each input variable on the curing degree can be analyzed to determine the optimal preheated temperature. Because of the small ranges of data, the calculation results do not change significantly, so the minimum spread value cannot be set as the optimal value during prediction. Thus the reverse design based on the curing degree is made to analyze the influence of the curing degree on the preheated temperature at different stages.

3) Due to the limited quantity and quality of samples, the prediction accuracy of RBF model based on these samples is surely affected. In order to analyze more accurately of the relationship between the curing degree and temperature in the pultrusion process of a GFRP pipe, statistical indicators are adopted in this paper to indicate that the prediction method is correct and feasible.

\section{References}

[1] A. Jacob. Globalization of the pultrusion industry [J]. Reinforced Plastics, 2006, 50 (5): 38-41.

[2] J. Martin. Pultruded composites compete with traditional construction materials [J]. Reinforced Plastics, 2006, 50 (5): 20-27.

[3] Eyassu W, Samuel K, Pang S. Optimization for buckling loads of grid stiffened composite panels [J]. Composite Structures, 2003, (60): 159-169.

[4] Jianhua Li, Sunil, C. Joshi etc. Curing Optimization for Pultruded Composite Sections. Composite Science and Technology [J], 2002, (62): 456-467.

[5] Lin He, Chuanzhen Huang. Artificial neural network and optimization method combination used in composite materials [C]. Silicate Bulletin, 2004, (1): 85.

[6] Huaiqin Xie, Xingkai Chen. Unsteady temperature field, numerical simulation of curing degree and experiment of GFRP pultrusion process [J]. GFRP /Composites, 2010, (1): 73-76. 